www.jkns.or.kr

\section{Case Report}

Seong Rok Han, M.D.

Chan Young Choi, M.D., Ph.D.

Mee Joo, M.D., Ph.D. 2

Choong Jin Whang, M.D.,

Ph.D., F.A.C.S. ${ }^{1}$

Departments of Neurosurgery ${ }^{l}$ Pathology, Ilsan Paik Hospital College of Medicine

Inje University, Goyang, Korea

\title{
Isolated Cerebral Mucormycosis
}

Isolated cerebral mucoromycosis, without rhino-orbital focus, is an extremely rare but life-threatening infection of central nervous system that most commonly found in intravenous drug abuser. We present a case of isolated cerebral mucormycosis diagnosed by open biopsy and treated with amphotericin B. The patient has returned to independent living.

KEY WORDS : Isolated · Cerebral Mucormycosis · Amphotericin B.

\section{INTRODUCTION}

Infection with fungi of the Mucoraceae family is rare, which is unfortunately, frequently fatal ${ }^{11)}$. Multiple clinical syndromes including rhinocerebral, pulmonary, gastrointestinal, cutaneous and disseminated forms have been described ${ }^{4,9,13)}$. Most mucormycosis cases are rhinocerebral in which the infection ascends from the nasal passage to sinuses or orbit and then sometimes to the brain ${ }^{5,10,13)}$. Open head injury can also implant this fungus directly into the brain ${ }^{12)}$. Isolated cerebral mucormycosis is a rarely encountered fungal infection seen in immunocompromised patients or patients with a history of intravenous drug abuse (IVDA) ${ }^{6}$. A literature search by Verma et $\mathrm{al}^{12}{ }^{12}$. reported 30 cases of isolated cerebral mucormycosis, which are associated with a history of IVDA. We present a case of isolated cerebral mucormycosis diagnosed by open biopsy and treated with amphotericin $\mathrm{B}$, with radiographic and clinical remission.

\section{CASE REPORT}

This 39-year-old woman with a history of insulin dependent diabetes mellitus was admitted to our hospital with a 10-day history of diplopia. The family members reported that the patient had complained of headache, amnesia and dizziness a few months ago. General physical examination was normal. There was no evidence of nasal inflammation or infections. Neurological examination was normal except that visual field examination showed right upper homonymous quadrantanopsia. There was no history of IVDA and head trauma. Laboratory findings showed a white blood cell count of $5,150 / \mathrm{mm}^{3}$ with $53 \%$ neutrophils, hemoglobin of $11.4 \mathrm{~g} / \mathrm{dL}$, hematocrit of $32 \%$, and platelet count of $212,000 / \mathrm{mm}^{3}$. Routine blood chemistry values were within normal limit except hyperglycemia $(447 \mathrm{mg} / \mathrm{dL})$. Radiographs of chest and sinuses were normal. Magnetic resonance (MR) images of brain showed that two irregular shaped lesions, which were located left thalamus and suprasellar area. Gadolinium enhanced MR images revealed that irregular rim enhancement of lesions (Fig. 1). A lumbar puncture yielded $0 \mathrm{RBC} / \mathrm{mm}^{3}$, $181 \mathrm{WBCs} / \mathrm{mm}^{3}$, with $100 \%$ lymphocytes, protein value 47 $\mathrm{mg} / \mathrm{dL}$, and glucose value 107 $\mathrm{mg} / \mathrm{dL}$. Gram's stain showed no organisms.

Open biopsy was performed on the suprasellar area via frontotemporal craniotomy.
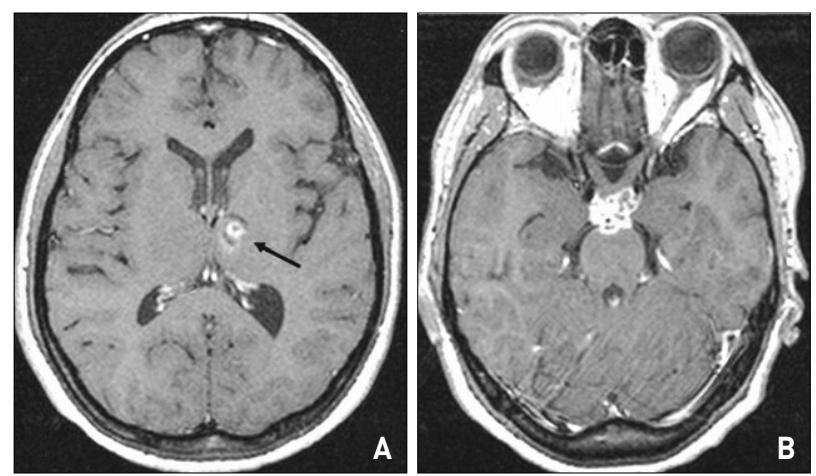

Fig. 1. Gadolinium enhanced axial T1-weighted magnetic resonance images show that two irregular enhancing lesions are located in the left thalamus $(A$, arrow) and suprasellar area $(B)$. 

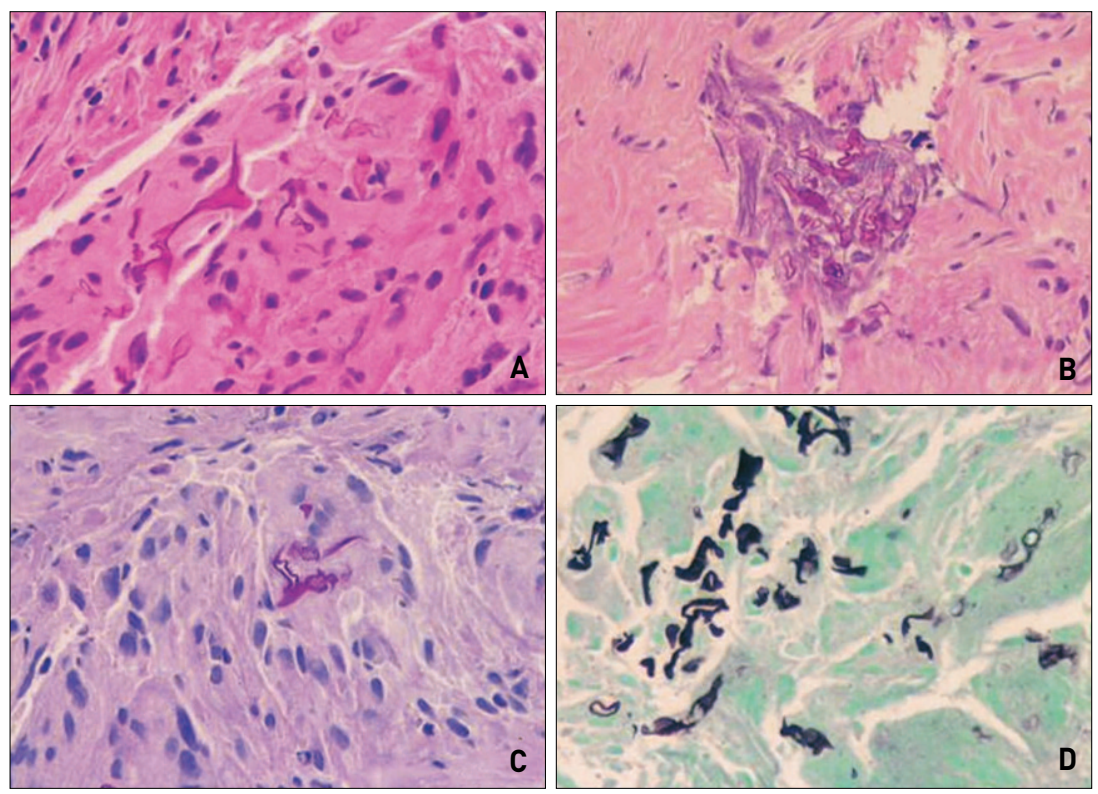

Fig. 2. Microphotographs $(A, B)$ showing many hyphae with irregular widths and right-angle branching are noted in the necrotic tissue as well as within the multinucleated giant cells ( $A$ : H\&E $\times 200 ; B: H \& E \times 400)$. PAS $(C: \times 400)$ and GMS $(D: \times 400)$ stains highlight fungal hyphae also.

\section{DISCUSSION}

Mucormycosis is a fungal infection most often develops in individuals with immunologically compromising conditions $^{1,8,12,13)}$. Risk factors contributing to CNS mucormycosis are diabetes mellitus, malignancy, and chronic immunosuppression ${ }^{12,13)}$. Particularly, when diabetes mellitus is poorly controlled that causes acidosis, it becomes a high risk factor as it relates to cellular immune dysfunction $^{4}$. Isolated cerebral mucormycosis with no evidence of regional or systemic disease is a very uncommon entity and IVDA history is the most important risk factor for isolated cerebral mucormycosis $^{2,12)}$.

The invasion pattern of cerebral
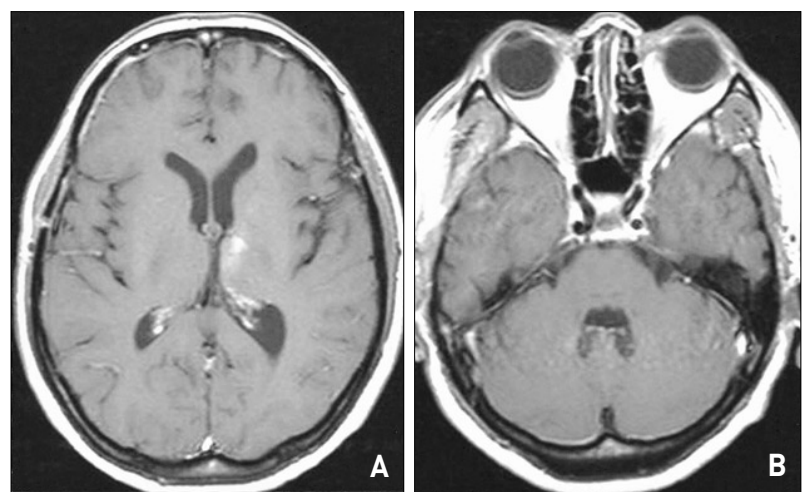

Fig. 3. Follow-up gadolinium enhanced axial T1-weighted magnetic resonance images after three months. There is markedly decreased pathologic enhancement in left thalamus (A) and suprasellar area (B).

Right opticcarotid space was widened with fibrosis. Underneath the optic nerve, dark grayish material tissue was noted. Pathologic examination revealed inflammation with epithelioid granulomas, neutrophils, lymphocytes, scattered multinucleated giant cells, and wide nonseptate hyphal fragments typical of the family Mucoraceae (Fig. 2). All fungal cultures were negative. Histologic findings were consistent with cerebral mucormycosis.

Treatment with intravenous amphotericin B $(1 \mathrm{mg} / \mathrm{kg} / \mathrm{d})$ was initiated. The patient's hyperglycemia was controlled with insulin. For the period of 15 weeks of therapy, the patient received an accumulated does of $5.423 \mathrm{~g}$ of amphotericin B without complication. Follow-up MR images showed gradual reduction in the size of cerebral lesions (Fig. 3). The patient was discharged from the hospital and returned to independent living. mucomycosis is somewhat predictable and may occur by direct invasion or hematogenous spread ${ }^{9)}$. The primary route of rhinocerebral mucormycosis is through direct extension from the nose and/or paranasal sinuses, underscoring the organism's predilection for the nasal cavity and paranasal sinuses. This is followed or accompanied by subsequent spread to the orbits after crossing bony partitions ${ }^{7}$. Invasion into the orbit causes orbital cellulitis, proptosis, opthalmoplegia and eventually blindness ${ }^{8,10)}$. The pathogens are prone to invade and spread along blood vessels, particularly arteries. The fungus proliferates within the internal elastic lamina, dissecting it away from the media. As the hyphae penetrate the endothelium, thrombotic arteritis, infarction, hemorrhage, and extensive necrosis follow ${ }^{13)}$.

The diagnosis is based on a clinical picture revealing the invasive course of the disease and is confirmed by biopsy, where the specimen usually show broad, non-septate hyphae with branching at right angles and invading tissue. Culture can be used to identify the specific species although the cultures may fail to grow despite the presence of widespread and aggressive disease $\mathrm{e}^{10)}$. Treatment consists of systemic antifungal therapy, radical surgical debridement and correction of any underlying condition ${ }^{3,8)}$. Systemic antifungal chemotherapy, limited to amphotericin B, has significantly improved the survival rates of patients with mucormycosis ${ }^{13}$. Therapeutic response to antifungal therapy alone is unreliable. Surgical procedures play a role in diagnosis, early and, if require, repeated surgery has been necessary to treat extensive disease ${ }^{13)}$. The case reported herein deserves attention because it was associated with two lesions in the cerebrum, no history 
of IVDA, negative for human immunodeficiency virus, and successful treatment result. Diabetes mellitus may have also been associated with this rare disorder in this patient.

\section{CONCLUSION}

Isolated cerebral mucormycosis with no evidence of regional or systemic disease is a very uncommon clinical entity. This entity is different from the rhinocerebral mucormycosis. Early diagnosis, surgical removal of devitalized infected tissue, intravenous amphotericin B therapy, and correction of any underlying condition represent a viable treatment option for this rare disorder.

\section{References}

1. Alleyne CH Jr, Vishteh AG, Spetzler RF, Detwiler PW : Long-term survival of a patient with invasive cranial base rhinocerebral mucormycosis treated with combined endovascular, surgical, and medical therapies : case report. Neurosurgery $45: 1461-1464,1999$

2. Blazquez R, Pinedo A, Cosin J, Miralles P, Lacruz C, Bouza E : Nonsurgical cure of isolated cerebral mucormycosis in an intravenous drug user. Eur J Clin Microbiol Infect Dis 15 : 598-599, 1996

3. Blitzer A, Lawson W, Meyers BR, Biller HF : Patient survival factors in paranasal sinus mucormycosis. Laryngoscope 90 : 635-648, 1980

4. Cheema SA, Amin F : Five cases of rhinocerebral mucormycosis : $\mathrm{Br}$ J Oral maxillofac Surg $12: 2-3,2005$

5. Escobar A, Del Brutto OH : Multiple brain abscesses from isolated cerebral mucormycosis. J Neurol Neurosurg Psychiatry 53 : 431433,1990

6. Gollard R, Rabb C, Larsen R, Chandrasoma P : Isolated cerebral mucormycosis : case report and therapeutic considerations. Neurosurgery $34: 174-177,1994$

7. Hamilton JF, Bartkowski HB, Rock JP : Management of CNS mucormycosis in the pediatric patient. Pediatr Neurosurg $38: 212-$ 215,2003

8. Kwak SW, Kim JT, Chung DS : Massive cerebral infarction due to rhinocerebral mucormycosis. J Korean Neurosurg Soc 39 : 455458, 2006

9. Orguc S, Yuceturk AV, Demir MA, Goktan C : Rhinocerebral mucormycosis : perineural spread via the trigeminal nerve. J Clin Neurosci 12 : 484-486, 2005

10. Ruoppi P, Dietz A, Nikanne E, Seppa J, Markkanen H, Nuutinen J : Paranasal sinus mucormycosis : a report of two cases. Acta Otolaryngol $121: 948-952,2001$

11. Siddiqi SU, Freedman JD : Isolated central nervous system mucormycosis : South Med J 87 : 997-1000, 1994

12. Verma A, Brozman B, Petito CK : Isolated cerebral mucormycosis : report of a case and review of the literature. J Neurol Sci $240: 65-$ 69, 2006

13. Weprin BE, Hall WA, Goodman J, Adams GL : Long-term survival in rhinocerebral mucormycosis : Case report. J Neurosurg $88: 570$ 575,1998 